\title{
24. Two-dimensional Brownian Motion and the Type Problem of Riemann Surfaces.
}

\author{
By Shizio KAKUTANI.
}

Mathematical Institute, Osaka Imperial University.

(Comm. by T. TAKAGI, M.I.A., March 12, 1945.)

1. Let $\Sigma$ be a simply connected, infinitely sheeted open Riemann surface.") Thein, as is well known, there exists an analytic function defined on $\Sigma$ which maps $\Sigma$ conformally onto the unit circle or onto the entrre Gaussian plane. The type of $\boldsymbol{\Sigma}$ is hyperbolic or paiabolic according as the first or the second case happens. It is an important problem in the theory of analytic functions to determine the type of such Riemann surfaces, and many necessary or sufficient conditions for each of these cases were obtained by several authors. But necessary and sufficient conditions are known only for some special classes of Riemann surfaces. The purpose of this paper is to give a necessary and sufficient condition in terms of Brownian motion on a Riemann surface.

2. In a previous paper ${ }^{2}$ we have discussed Brownian motion on the Gaussian plane and have obtained the following theorem: Let $D$ be a Jordan domain in the Gaussian plane and let $\boldsymbol{E}$ be an arbitrary elementary set on the boundary $\Gamma=B d(D)$ of $D$. Then the probability $P(\zeta, E, D)$ that the Brownian motion starting from a point $\zeta \in D$ will enter into $E$ without entering into the other part $\Gamma-E$ of the boundary of $D$ before it (i.e. without getting out of $D$ before $i t$ ), is a bounded, harmonic function of $\zeta$ on $D$ which tends to 1 or to 0 according as $\zeta$ tends to an inner point of $E$ or of $\Gamma-E$.

In order to apply this result to our problem, let us first notice that it is $\mathrm{m}$ necessary to assume that $D$ is one-sheetcl. $D$ cim, in fact, be any finitely shected Jordan domain on a Riemann surface hiving; no algebraic branch points in it. This follows from the fact that $P(\zeta, E, I)$ ) is a function of $\zeta$ defined on a multiply sheeted domain $D$; satisfying the boundary conditions stated above, which is locally harmonic on $D$. It is of course unilerstood that the Brownian motion is defined on the multiply sheeted domain $D$, so that a path having double points on the Gaussian plane may have no double points on $D$. The condition thiat $D$ is bounded is also unnecessary.

1. As for the type problem of Riemann surfaces, see R. Nevanlinna, Eindeutige analytische Funktionen, 1936, Chapter XIII.

2. S. Kakutani, Two-dimensional Browrian motion and harmonic functions, Proc. 20 (1944), 700-708. 
No. 3.] Twc-dimensional Brownian Motion and the Type Problem of Riemann Surfaces. 139

Next we notice that the existence of a finite number of algebraic branch points $A=\left\{\alpha_{1}, \ldots, \alpha_{p}\right\}$ in $D$ does not afiect the validity of our theorem if we aclopt the following convention: if $\alpha$ is an algebraic branch point of $D$, then $P(\alpha, E, D)$ is defined as the limit of $P(\zeta, E, D)$ as $\zeta$ tends to $\alpha$ on the multiply sheeted domain $D$. This follows from the following two facts: (i) for any $\zeta \in D-A$, the probalility that the Brownian motion on $D$ starting from s will hit at an algebraic branch point of $D$ (without getting out of $D$ before it) is zero, (ii) $P(\zeta, E, D)$ is a bounded harmonic function defined on $D-A$ and every algebraic branch point of $D$ is a removable singularity of $P(\zeta, E, D)$.

Thus $D$ ean be any finitely sheeted Jordan domain on a Piemann surface, bounded or not, having a finite number of algebraic branch points in it. Such a domain $D$ will be called a Riemann-Jordan domain.

3. Let $\Sigma$ be a simply connected, infinitely sheeted open Riemann surface. We assume that $\Sigma$ is given as the union of an increasing sequence of Riemann Jordan domains $\left\{D_{n} \mid n=1,2, \ldots\right\}$ stch that $\bar{D}_{n} \subset D_{n+1}, n=1,2, \ldots$ This means that $D_{n}$ is a subdomain of $D_{n+1}$, both being considered as multiply sheeted clomains, and that the boundary $\Gamma_{n}=B a\left(D_{n}\right)$ of $D_{n}$ is contained in $D_{n+1}$.

Let $D_{0}$ be a simply connecfed Riemann-Jordan domain on $\boldsymbol{\Sigma}$ with the boundary $\Gamma_{0}=B d\left(D_{0}\right)$, so that $\boldsymbol{\Sigma}-\bar{D}_{0}$ is a doubly connected subdomain of $\boldsymbol{\Sigma}$ whose boundary relative to $\Sigma$ is exactly $\Gamma_{0}$. For example, take as $D$ a small one-sheeted circular domain on $\boldsymbol{\Sigma}$ having no algebraic branch points of $\boldsymbol{\Sigma}$ in it. We may assume that $\bar{D}_{0} \subset D_{\mathrm{i}}$.

For any $n \geq 1$ and for any $\zeta \in D_{n}-\overline{D_{0}}$, let $u_{n}(\zeta)=P\left(\zeta, \Gamma_{0}, D_{n}-\overline{D_{0}}\right)$ be the probability that the Brownian motion starting from $\zeta$ will enter into $\Gamma_{0}$ without entering into $\Gamma_{n}=B d\left(D_{n}\right)$ before it. Then $u_{n}(\zeta)$ is a bounded harmonic function defined on $D_{n}-\bar{D}_{0}$ which tends to 1 or to 0 according as $\zeta$ tends to a point on $\Gamma_{0}$ or on $\Gamma_{n}$.

It is clear that $u_{n}(\zeta) \leqq u_{n+1}(\zeta)$ on $D_{n}-\bar{D}_{0}, n=1,2, \ldots$ Hence $\lim _{n \rightarrow \infty}$ $u_{n}(\zeta)=u(\zeta)$ exists and is harmonic on $\Sigma-\bar{D}_{0}=\bigcup_{n=1}^{\infty}\left(D_{n}-\bar{D}_{0}\right) . \quad u(\zeta)$ is the probability that the Brownian motion on $\Sigma$ starting from $\zeta$ will enter into $\Gamma_{0}=$ $B d\left(D_{0}\right)$ (without getting out of $\Sigma-\bar{D}_{0}$ before it). We shalledenote this probal ility by $P\left(\zeta, \Gamma_{0}, \Sigma-\overline{D_{0}}\right)$. Then our main result may be stated as follows:

Theorem. $u(\zeta)=P\left(\zeta, \Gamma_{0}, \Sigma-\bar{D}_{0}\right)$ is either $<1$ throughout on $\Sigma-\bar{D}_{0}$ or $\equiv 1$ on $\boldsymbol{\Sigma}-\overline{D_{0}}$ according as $\boldsymbol{\Sigma}$ is of hyperbolic or of paralolic type.

Proof. Let $\zeta_{0} \in D_{0}$ be an arbitrary point. Let $\omega=\varphi(\zeta)$ be an analytic function satisfying $0=\boldsymbol{\phi}\left(\zeta_{0}\right)$ which' maps $\Sigma$ conformally onto the unit circle $\Sigma^{\prime}$ $=\{\omega|| \omega \mid<1\}$ or onto the entire Gaussian plane $\Sigma^{\prime}=\{\omega|| \omega \mid<\infty\}$ according 
as $\boldsymbol{I}$ is hyperbolic or paral olic. Let us put $I^{\prime}{ }_{n}=\phi\left(D_{n}\right), \Gamma_{n}^{\prime}=\varphi\left(I_{n}^{\prime}\right), n=0$, $1,2, \ldots$ Then each $\Gamma^{\prime}{ }_{n}(n \geqq 0)$ is a simple closed Jordan curve which separates $\omega=0$ from $\omega=\infty$. It is clear that $\Sigma^{\prime \prime}=\bigcup_{n=1}^{\infty} J^{\prime}{ }_{n}$. Further, $u_{n}^{\prime}(\omega)=u_{n}\left(\varphi^{-1}(\omega)\right)$ is a bounded harmonic function (lefined on $D^{\prime}{ }_{n}-\overline{J)_{0}^{\prime}}$ which tends to 1 or to 0 according as $\omega$ tends to a point on $\Gamma_{0}^{\prime}$ or on $\Gamma_{n}^{\prime}$. Thus $u_{n}^{\prime}(\omega)$ is nothing else than the probalility $\left.P\left(\omega, \Gamma_{0}^{\prime}, I\right)^{\prime}{ }_{n}-\bar{I}_{11}^{\prime}\right)$ that the Brownian motion on the Gaussian plane starting from $\omega$ will enter into $\Gamma_{0}^{\prime}$ without entering into $\Gamma_{n}^{\prime}$ before it. It is clear that $u_{n}^{\prime}(\omega) \leqq u_{n+1}^{\prime}(\omega)$ on $D_{n}^{\prime}-\bar{D}_{0}^{\prime}, n=1,2, \ldots$ Hence $u^{\prime}(\omega)=$ $\lim _{n \rightarrow \infty} u^{\prime}{ }_{n}(\omega)$ exists on $\left.\Sigma^{\prime}-I\right)_{0}^{\prime}=\bigcup_{n=1}^{\infty}\left(D_{n}^{\prime}-\overline{I_{n}^{\prime}}\right)$ and the limit $u^{\prime}(\omega)$ is equal to the probability $P\left(\omega, \Gamma_{0}^{\prime}, \Sigma^{\prime}-\bar{D}_{0}^{\prime}\right)$ that the Brownian motion on the Gaussian plane starting from $\omega$ will enter into $\Gamma^{\prime}{ }_{0}$ without getting out of $\Sigma^{\prime}$ before it. If $\boldsymbol{\Sigma}$ is hyperbolic (i.e. if $\mathbf{S}^{\prime}$ is the unit (ircle) then this probability is $<1$ throughout on $\Sigma^{\prime}-\bar{D}_{0}^{\prime}$, while it is $\equiv \mathbf{1}$ on $\left.\Sigma^{\prime}-I\right)_{0}^{\prime}$ if $\boldsymbol{\Sigma}$ is parabolic (i.e. if $\Sigma^{\prime}$ is the entire Gaussian planc). The proof of our theorem is completed if we observe that $u\left(\varphi^{-1}(\omega)\right) \equiv I^{\prime}\left(\phi^{-1}(\omega), \Gamma_{0}, \mathbf{\Sigma}-\bar{D}_{0}\right)=u^{\prime}(\omega) \equiv P\left(\omega, \Gamma_{0}^{\prime}, \Sigma^{\prime}-\bar{D}_{i j}^{\prime}\right)$. 